\title{
Scanning Auger for Defect Root Cause Analysis: Advantages \& Application Challenges
}

\author{
Y.S. Uritsky, A. Anapolsky \\ Defect and Thin Film Characterization Laboratory, \\ Applied Materials, Inc., Santa Clara, California 95054, USA
}

Development of future semiconductor processes and improving existing ones requires a strong capability to identify and eliminate defects - the major yield detractor of IC manufacturing. The current industry approach for accomplishing this task is to analyze them on the wafer surface. The most commonly used technique for this purpose is SEM with EDX (SEM/EDX). This approach provides information about defect chemistry and morphology, which in turn provides a direct path to the re-evaluation of processes and hardware parts needed to determine the root cause of a problem. However, the SEM/EDX has two fundamental limitations: (a) it is a large volume technique and therefore, the sensitivity can be insufficient for sub micron particles and thin patches; (b) there is no chemical bonding information due to poor energy resolution. In many cases there is a need to use other techniques to support or substitute EDX. The most suitable technique for this purpose is Scanning Auger Microscope (SAM), e.g. Smart-300 (integrated SAM/EDX/FIB instrument) made by PHI. Auger typically gives information on approximately the top 10 atomic layers, with the signal decaying exponentially with depth. For particles on surfaces the situation is complex, because the high energy primary beam (typically $5-20 \mathrm{KeV}$ ) can scatter laterally out of the particle and strike the adjacent substrate surface producing a component in the observed Auger spectrum which does not come from the particle. Thus, even a primary beam as small as $150 \mathrm{~A}$ will not guarantee that the Auger signal comes entirely from the area of interest. Correct spectra interpretation becomes even more difficult when in addition to scattering phenomena the energy peaks of different elements are too close to each (line overlap problems). We faced this problem when SAM was used for analysis of wafers after chemical mechanical polishing (CMP) process. Specifically, the analyzed wafer presented W plugs with TiN layer in-between. CMP process materials contained some amount of tin; hence the analysis was focused on mapping of $\mathrm{W}$, Ti, and Sn.

Results are presented in Figure 1(a,b), which illustrate what kinds of precautions are needed for proper mapping of Ti and Sn. In addition to very high surface sensitivity, the Auger technique has some chemical state analysis capabilities, in principle available from chemical shift information. This capability was used for particle root cause analysis. Specifically, by analyzing the particle cross-section (FIB cut) it was shown that the particle's core is $\mathrm{Al}$ metal, not $\mathrm{Al}^{3+}$ state (characteristic of oxide or fluoride). Subsequently, obtained information resulted in the correct identification of the source of particles - it was degraded 'face plate' made of Al alloy (see AlKLL spectra with exact $16 \mathrm{eV}$ between the plasmon losses peaks (prove that it is indeed an Al metal) shown in Figure 2).

Described examples, as well as an application development given in [1] have led to conclusion that combination of fast SEM/EDX with slower but more analytically capable SAM is now the most effective approach for defect characterization.

References: [1] Childs, K.D., et al., Analysis of Submicron Defects Using an SEM-Auger Defect Review Tool, Characterization and Metrology for ULSI Technology, AIP CP 449, pp.810-814. 

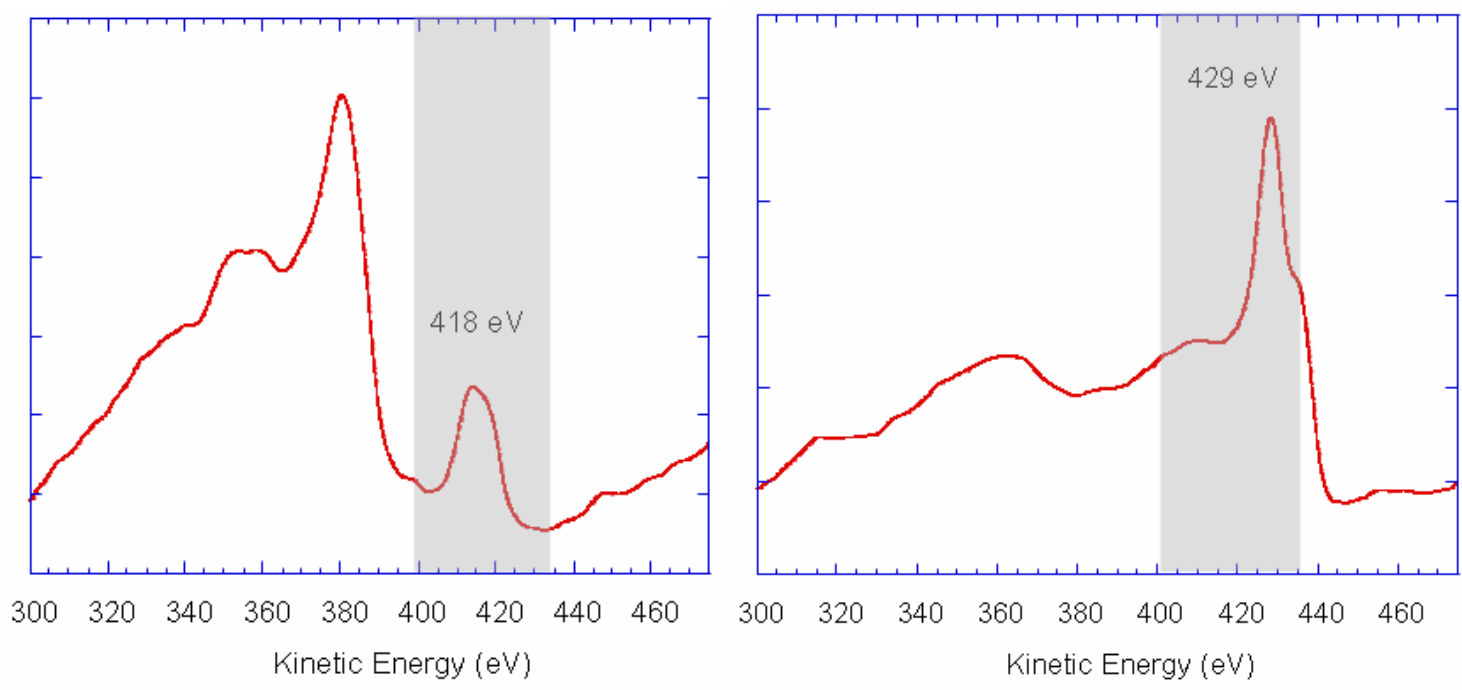

Figure 1(a). Auger spectra taken from Ti (left) and Sn (right), Geller's standards. Gray areas indicate the overlap of primary $\mathrm{Ti}$ and $\mathrm{Sn}$ Auger peaks.
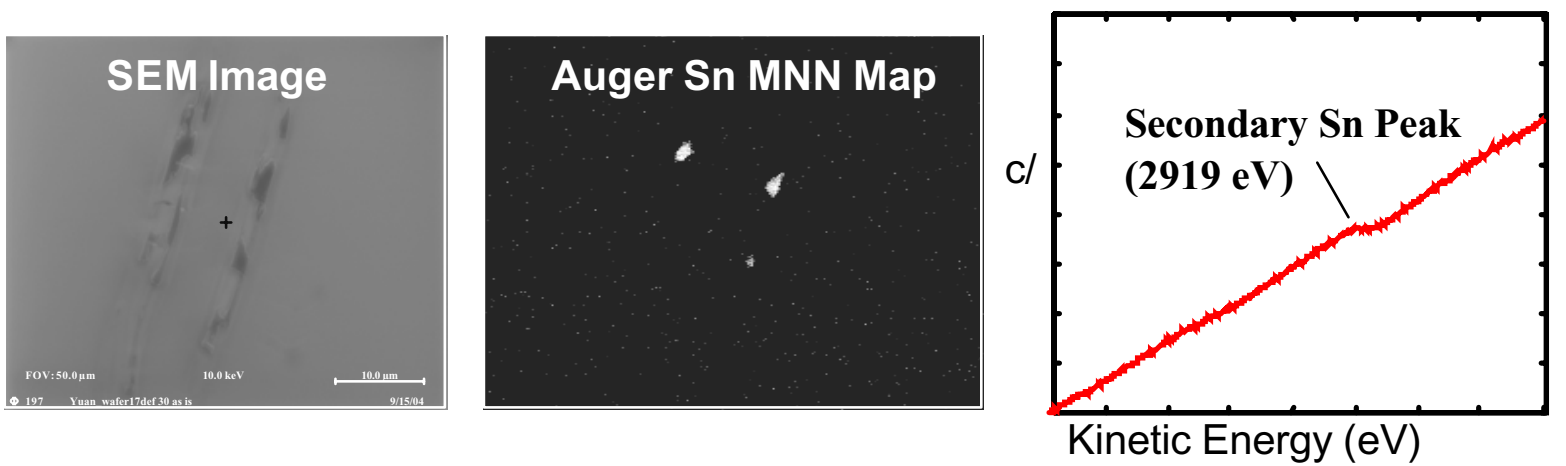

Figure 1(b). Wafer region with CMP defects (left - SEM, center - Sn Auger Map, right - the solution used to resolve this overlap). Detailed Auger analysis showed that these defects were Tibased. Use of the Sn secondary $(2919 \mathrm{eV})$ peak has resolved this problem.
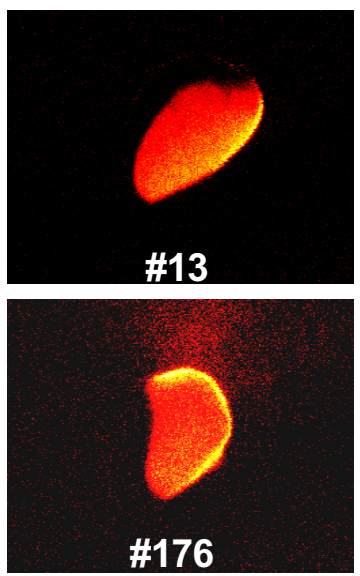
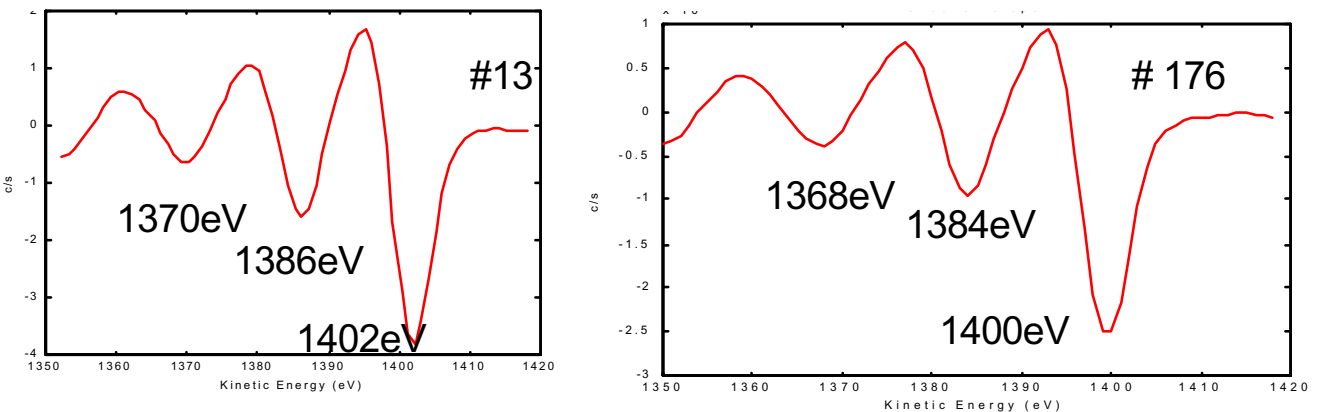

Figure 2. Al Elemental Auger Map (left) and High Resolution Al KLL spectra of Particles \#\# 13 \& 176 taken after FIB X-sectioning. Difference between the plasmon losses peaks is $16 \mathrm{eV}$ ( $\mathrm{Al}$ metal). 